\title{
Interaction of Two Pulsatory Waves of the Korteweg-de Vries Equation in a Zigzag Hyperbolic Structure
}

\author{
Victor A. Miroshnikov \\ Department of Mathematics, College of Mount Saint Vincent, New York, USA \\ Email: victor.miroshnikov@mountsaintvincent.edu
}

Received 5 May 2014; revised 2 June 2014; accepted 8 June 2014

Copyright (C) 2014 by author and Scientific Research Publishing Inc.

This work is licensed under the Creative Commons Attribution International License (CC BY).

http://creativecommons.org/licenses/by/4.0/

c) (i) Open Access

\begin{abstract}
A new exact solution for nonlinear interaction of two pulsatory waves of the Korteweg-de Vries (KdV) equation is computed by decomposition in an invariant zigzag hyperbolic tangent (ZHT) structure. A computational algorithm is developed by experimental programming with lists of equations and expressions. The structural solution is proved by theoretical programming with symbolic general terms. Convergence, tolerance, and summation of the ZHT structural approximation are discussed. When a reference level vanishes, the two-wave solution is reduced to the two-soliton solution of the KdV equation.
\end{abstract}

\section{Keywords}

KdV Equation, Two Pulsatory Waves, ZHT Structure, Experimental, Theoretical Computation

\section{Introduction}

Since the discovery of the $\mathrm{N}$-soliton solution of the Korteweg-de Vries (KdV) equation by experimental computation [1], various analytical methods, like the inverse scattering transform, the Bäcklund transform, generalized functions, etc. [2]-[5], were developed and implemented by theoretical computation [6]. Further developments of the theory of solitons comprise effects of vorticity [7]-[9] and viscosity [10], while computational methods evolved from asymptotic methods [11]-[13], to series approximations [14]-[16], and structural decompositions in invariant structures [17] [18]. In the current paper, an invariant zigzag hyperbolic-tangent (ZHT) structure is developed to treat nonlinear interaction of two pulsatory waves of the KdV equation. A zigzagging pattern is a ubiquitous phenomenon in fluid-dynamic, biological, and chemical systems [19]-[21].

The structure of this paper is as follows. In section 2, experimental computation with lists of equations and 
expression is used to develop a computational algorithm in Maple ${ }^{\mathrm{TM}}$ and explore the convergence of the ZHT structural approximation. Theoretical computation with symbolic general terms is utilized in Section 3 to develop differentiation and multiplication formulas for the ZHT structure, show its invariance, and consider summation, convergence and tolerance of the structural approximation. The two-wave solution is visualized and compared with the two-soliton solution in Section 4, which is followed by a summary of main results in Section 5.

\section{Experiment on Interaction of Two Pulsatory Waves in the ZHT Structure}

\subsection{Formulation of an Experimental Problem in the ZHT Structure}

Explore experimentally a structural solution of the canonical form of the $\mathrm{KdV}$ equation

$$
\frac{\partial \phi}{\partial t}+6 \phi \frac{\partial \phi}{\partial x}+\frac{\partial^{3} \phi}{\partial x^{3}}=0
$$

for nonlinear interaction of two pulsatory waves. Construct the structural solution in the ZHT structure of algebraic order $M=3$, which is used to illustrate a computational algorithm,

$$
\phi(x, t)=h+2 p^{2} \mu^{2}\left[Z_{0,0}+Z_{2,0} t a^{2}+\left(Z_{1,1} t a+Z_{3,1} t a^{3}\right) T b+\left(Z_{2,2} t a^{2}+Z_{4,2} t a^{4}\right) T b^{2}+\left(Z_{3,3} t a^{3}+Z_{5,3} t a^{5}\right) T b^{3}\right],
$$

where $h$ is a reference level, $Z_{m+2, m}$ and $Z_{m, m}$ are structural coefficients, $t a=\tanh (\mu X)$ and $T b=q t b=\tanh (v Y)$ are structural functions, $\mu=\frac{1}{2} \sqrt{U-6 h}$ and $v=\frac{1}{2} \sqrt{V-6 h}$ are wave numbers such that $0 \leq v<\mu, X=x-U t-a$ and $Y=x-V t-b$ are propagation variables, $U$ and $V$ are celerities, $a$ and $b$ are initial locations of first and second pulsatory waves, $q=\frac{v}{\mu}$ and $p=\sqrt{1-q^{2}}$ are interaction and complementary parameters such that $0 \leq q<1$ and $0<p \leq 1$, respectively. When $p=1(q=0), Z_{0,0}=1$, and $Z_{0,2}=-p^{2}$, the two-wave solution is reduced to a one-wave solution

$$
\begin{aligned}
\phi(x, t) & =h+2 \mu^{2}\left(1-t a^{2}\right) \\
& =h+\frac{1}{2}(U-6 h) \operatorname{sech}^{2}\left(\frac{1}{2} \sqrt{U-6 h}(x-U t-a)\right),
\end{aligned}
$$

which becomes the one-soliton solution $\phi(x, t)=\frac{U}{2} \operatorname{sech}^{2}\left(\frac{1}{2} \sqrt{U}(x-U t-a)\right)$ [3] for $h=0$. Consider an instance of (2) with $h=1 / 8, U=3, V=1, q=1 / 3, p=2 \sqrt{2} / 3, \mu=3 / 4$, and $v=1 / 4$. The ZHT structure then is

$$
\begin{aligned}
\phi(x, t)= & \frac{1}{8}+Z_{0,0}+Z_{2,0} t a^{2}+\left(Z_{1,1} t a+Z_{3,1} t a^{3}\right) T b \\
& +\left(Z_{2,2} t a^{2}+Z_{4,2} t a^{4}\right) T b^{2}+\left(Z_{3,3} t a^{3}+Z_{5,3} t a^{5}\right) T b^{3} .
\end{aligned}
$$

\subsection{Experimental Differentiation of the ZHT Structure}

Primarily, symbolic computation of a spatial derivative of differential order $N=1$ of two-wave solution (4) yields the ZHT structure of algebraic order $M+N=4$

$$
\begin{aligned}
\frac{\partial \phi}{\partial x}= & A_{1,0} t a+A_{3,0} t a^{3}+\left(A_{0,1}+A_{2,1} t a^{2}+A_{4,1} t a^{4}\right) T b \\
& +\left(A_{1,2} t a+A_{3,2} t a^{3}+A_{5,2} t a^{5}\right) T b^{2} \\
& +\left(A_{2,3} t a^{2}+A_{4,3} t a^{4}+A_{6,3} t a^{6}\right) T b^{3}+\left(A_{3,4} t a^{3}+A_{5,4} t a^{5}\right) T b^{4},
\end{aligned}
$$

with a general term $A_{n, m} t a^{n} T b^{m}$, where structural coefficients $A_{n, m}$ are 


$$
\begin{aligned}
& A_{1,0}=\frac{3}{2} Z_{2,0}+\frac{1}{12} Z_{1,1}, A_{3,0}=-\frac{3}{2} Z_{2,0}+\frac{1}{12} Z_{3,1}, \\
& A_{0,1}=\frac{3}{4} Z_{1,1}, A_{2,1}=-\frac{3}{4} Z_{1,1}+\frac{9}{4} Z_{3,1}+\frac{1}{6} Z_{2,2}, \\
& A_{4,1}=-\frac{9}{4} Z_{3,1}+\frac{1}{6} Z_{4,2}, A_{1,2}=-\frac{3}{4} Z_{1,1}+\frac{3}{2} Z_{2,2}, \\
& A_{3,2}=-\frac{3}{4} Z_{3,1}-\frac{3}{2} Z_{2,2}+3 Z_{4,2}+\frac{1}{4} Z_{3,3}, A_{5,2}=-3 Z_{4,2}+\frac{1}{4} Z_{5,3}, \\
& A_{2,3}=-\frac{3}{2} Z_{2,2}+\frac{9}{4} Z_{3,3}, A_{4,3}=-\frac{3}{2} Z_{4,2}-\frac{9}{4} Z_{3,3}+\frac{15}{4} Z_{5,3}, \\
& A_{6,3}=-\frac{15}{4} Z_{5,3}, A_{3,4}=-\frac{9}{4} Z_{3,3}, A_{5,4}=-\frac{9}{4} Z_{5,3} .
\end{aligned}
$$

Secondly, computation of a temporal derivative of differential order $N=1$ also returns the ZHT structure of algebraic order $M+N=5$

$$
\begin{aligned}
\frac{\partial \phi}{\partial t}= & T_{1,0} t a+T_{3,0} t a^{3}+\left(T_{0,1}+T_{2,1} t a^{2}+T_{4,1} t a^{4}\right) T b \\
& +\left(T_{1,2} t a+T_{3,2} t a^{3}+T_{5,2} t a^{5}\right) T b^{2} \\
& +\left(T_{2,3} t a^{2}+T_{4,3} t a^{4}+T_{6,3} t a^{6}\right) T b^{3} \\
& +\left(T_{3,4} t a^{3}+T_{5,4} t a^{5}\right) T b^{4}
\end{aligned}
$$

with a general term $T_{n, m} t a^{n} T b^{m}$ and the following structural coefficients $T_{n, m}$ :

$$
\begin{aligned}
& T_{1,0}=-\frac{9}{2} Z_{2,0}-\frac{1}{12} Z_{1,1}, T_{3,0}=\frac{9}{2} Z_{2,0}-\frac{1}{12} Z_{3,1}, \\
& T_{0,1}=-\frac{9}{4} Z_{1,1}, T_{2,1}=\frac{9}{4} Z_{1,1}-\frac{27}{4} Z_{3,1}-\frac{1}{6} Z_{2,2}, \\
& T_{4,1}=\frac{27}{4} Z_{3,1}-\frac{1}{6} Z_{4,2}, T_{1,2}=\frac{3}{4} Z_{1,1}-\frac{9}{2} Z_{2,2}, \\
& T_{3,2}=\frac{3}{4} Z_{3,1}+\frac{9}{2} Z_{2,2}-9 Z_{4,2}-\frac{1}{4} Z_{3,3}, T_{5,2}=9 Z_{4,2}-\frac{1}{4} Z_{5,3}, \\
& T_{2,3}=\frac{3}{2} Z_{2,2}-\frac{27}{4} Z_{3,3}, T_{4,3}=\frac{3}{2} Z_{4,2}+\frac{27}{4} Z_{3,3}-\frac{45}{4} Z_{5,3}, \\
& T_{6,3}=\frac{45}{4} Z_{5,3}, T_{3,4}=\frac{9}{4} Z_{3,3}, T_{5,4}=\frac{9}{4} Z_{5,3} .
\end{aligned}
$$

Finally, a spatial derivative of order $N=3$ again produces the ZHT structure of order $M+N=6$

$$
\begin{aligned}
\frac{\partial^{3} \phi}{\partial x^{3}}= & B_{1,0} t a+B_{3,0} t a^{3}+B_{5,0} t a^{5}+\left(B_{0,1}+B_{2,1} t a^{2}+B_{4,1} t a^{4}+B_{6,1} t a^{6}\right) T b \\
& +\left(B_{1,2} t a+B_{3,2} t a^{3}+B_{5,2} t a^{5}+B_{7,2} t a^{7}\right) T b^{2} \\
& +\left(B_{0,3}+B_{2,3} t a^{2}+B_{4,3} t a^{4}+B_{6,3} t a^{6}+B_{8,3} t a^{8}\right) T b^{3} \\
& +\left(B_{1,4} t a+B_{3,4} t a^{3}+B_{5,4} t a^{5}+B_{7,4} t a^{7}\right) T b^{4} \\
& +\left(B_{2,5} t a^{2}+B_{4,5} t a^{4}+B_{6,5} t a^{6}\right) T b^{5} \\
& +\left(B_{3,6} t a^{3}+B_{5,6} t a^{5}\right) T b^{6}
\end{aligned}
$$

with a general term $B_{n, m} t a^{n} T b^{m}$, where structural coefficients $B_{n, m}$ become 


$$
\begin{aligned}
& B_{1,0}=-\frac{27}{4} Z_{2,0}-\frac{7}{24} Z_{1,1}+\frac{27}{32} Z_{3,1}+\frac{1}{16} Z_{2,2}, \\
& B_{3,0}=\frac{135}{8} Z_{2,0}+\frac{9}{32} Z_{1,1}-\frac{61}{24} Z_{3,1}-\frac{1}{16} Z_{2,2}+\frac{1}{8} Z_{4,2}+\frac{1}{288} Z_{3,3}, \\
& B_{5,0}=-\frac{81}{8} Z_{2,0}+\frac{27}{16} Z_{3,1}-\frac{1}{8} Z_{4,2}+\frac{1}{288} Z_{5,3} \text {, } \\
& B_{0,1}=-\frac{9}{8} Z_{1,1}+\frac{81}{32} Z_{3,1}+\frac{9}{16} Z_{2,2} \text {, } \\
& B_{2,1}=\frac{117}{32} Z_{1,1}-\frac{837}{32} Z_{3,1}-\frac{7}{3} Z_{2,2}+\frac{27}{8} Z_{4,2}+\frac{9}{32} Z_{3,3} \text {, } \\
& B_{4,1}=-\frac{81}{32} Z_{1,1}+\frac{783}{16} Z_{3,1}+\frac{27}{16} Z_{2,2}-\frac{109}{12} Z_{4,2}-\frac{9}{32} Z_{3,3}+\frac{15}{32} Z_{5,3} \text {, } \\
& B_{6,1}=-\frac{405}{16} Z_{3,1}+\frac{45}{8} Z_{4,2}-\frac{15}{32} Z_{5,3}, B_{1,2}=\frac{93}{32} Z_{1,1}-\frac{243}{32} Z_{3,1}-9 Z_{2,2}+\frac{81}{8} Z_{4,2}+\frac{81}{32} Z_{3,3} \text {, } \\
& B_{3,2}=-\frac{81}{32} Z_{1,1}+\frac{741}{32} Z_{3,1}+\frac{153}{8} Z_{2,2}-\frac{549}{8} Z_{4,2}-\frac{253}{32} Z_{3,3}+\frac{135}{16} Z_{5,3} \text {, } \\
& B_{5,2}=-\frac{243}{16} Z_{3,1}-\frac{81}{8} Z_{2,2}+\frac{873}{8} Z_{4,2}+\frac{81}{16} Z_{3,3}-\frac{685}{32} Z_{5,3} \text {, } \\
& B_{7,2}=-\frac{405}{8} Z_{4,2}+\frac{405}{32} Z_{5,3}, B_{0,3}=\frac{81}{32} Z_{1,1}-\frac{81}{16} Z_{2,2}+\frac{81}{32} Z_{3,3} \text {, } \\
& B_{2,3}=-\frac{81}{32} Z_{1,1}+\frac{243}{32} Z_{3,1}+\frac{177}{8} Z_{2,2}-\frac{243}{8} Z_{4,2}-\frac{1053}{32} Z_{3,3}+\frac{405}{16} Z_{5,3} \text {, } \\
& B_{4,3}=-\frac{243}{32} Z_{3,1}-\frac{243}{16} Z_{2,2}+\frac{663}{8} Z_{4,2}+\frac{891}{16} Z_{3,3}-\frac{2295}{16} Z_{5,3} \text {, } \\
& B_{6,3}=-\frac{405}{8} Z_{4,2}-\frac{405}{16} Z_{3,3}+\frac{6615}{32} Z_{5,3}, B_{8,3}=-\frac{2835}{32} Z_{5,3} \text {, } \\
& B_{1,4}=-\frac{81}{32} Z_{1,1}+\frac{243}{16} Z_{2,2}-\frac{729}{32} Z_{3,3}, B_{3,4}=-\frac{81}{32} Z_{3,1}-\frac{243}{16} Z_{2,2}+\frac{243}{8} Z_{4,2}+\frac{1179}{16} Z_{3,3}-\frac{1215}{16} Z_{5,3} \text {, } \\
& B_{5,4}=-\frac{243}{8} Z_{4,2}-\frac{729}{16} Z_{3,3}+\frac{3123}{16} Z_{5,3}, B_{7,4}=-\frac{3645}{32} Z_{5,3} \text {, } \\
& B_{2,5}=-\frac{81}{8} Z_{2,2}+\frac{729}{16} Z_{3,3} \text {, } \\
& B_{4,5}=-\frac{81}{8} Z_{4,2}-\frac{729}{16} Z_{3,3}+\frac{1215}{16} Z_{5,3}, B_{6,5}=-\frac{1215}{16} Z_{5,3} \text {, } \\
& B_{3,6}=-\frac{405}{16} Z_{3,3}, B_{5,6}=-\frac{405}{16} Z_{5,3} \text {. }
\end{aligned}
$$

\subsection{Experimental Multiplication of the ZHT Structures}

Symbolic computation of a product of two-wave solution (4) and first spatial derivative (5) once more produces the ZHT structure of algebraic order $2 M+N=7$

$$
\begin{aligned}
\phi \frac{\partial \phi}{\partial x}= & P_{1,0} t a+P_{3,0} t a^{3}+P_{5,0} t a^{5}+\left(P_{0,1}+P_{2,1} t a^{2}+P_{4,1} t a^{4}+P_{6,1} t a^{6}\right) T b \\
& +\left(P_{1,2} t a+P_{3,2} t a^{3}+P_{5,2} t a^{5}+P_{7,2} t a^{7}\right) T b^{2}+\left(P_{2,3} t a^{2}+P_{4,3} t a^{4}+P_{6,3} t a^{6}+P_{8,3} t a^{8}\right) T b^{3} \\
& +\left(P_{3,4} t a^{3}+P_{5,4} t a^{5}+P_{7,4} t a^{7}+P_{9,4} t a^{9}\right) T b^{4}+\left(P_{4,5} t a^{4}+P_{6,5} t a^{6}+P_{8,5} t a^{8}+P_{10,5} t a^{10}\right) T b^{5} \\
& +\left(P_{5,6} t a^{5}+P_{7,6} t a^{7}+P_{9,6} t a^{9}+P_{11,6} t a^{11}\right) T b^{6}+\left(P_{6,7} t a^{6}+P_{8,7} t a^{8}+P_{10,7} t a^{10}\right) T b^{7}
\end{aligned}
$$


with a general term $P_{n, m} t a^{n} T b^{m}$ and the following structural coefficients $P_{n, m}$ :

$$
\begin{aligned}
& P_{1,0}=\left(Z_{0,0}+\frac{1}{8}\right) A_{1,0}, P_{3,0}=\left(Z_{0,0}+\frac{1}{8}\right) A_{3,0}+Z_{2,0} A_{1,0}, \\
& P_{5,0}=Z_{2,0} A_{3,0}, P_{0,1}=\left(Z_{0,0}+\frac{1}{8}\right) A_{0,1} \text {, } \\
& P_{2,1}=\left(Z_{0,0}+\frac{1}{8}\right) A_{2,1}+Z_{2,0} A_{0,1}+Z_{1,1} A_{1,0}, \\
& P_{4,1}=\left(Z_{0,0}+\frac{1}{8}\right) A_{4,1}+Z_{2,0} A_{2,1}+Z_{3,1} A_{1,0}+Z_{1,1} A_{3,0}, \\
& P_{6,1}=Z_{3,1} A_{3,0}+Z_{2,0} A_{4,1}, P_{1,2}=\left(Z_{0,0}+\frac{1}{8}\right) A_{1,2}+Z_{1,1} A_{0,1} \text {, } \\
& P_{3,2}=\left(Z_{0,0}+\frac{1}{8}\right) A_{3,2}+Z_{2,0} A_{1,2}+Z_{3,1} A_{0,1}+Z_{1,1} A_{2,1}+Z_{2,2} A_{1,0} \text {, } \\
& P_{5,2}=\left(Z_{0,0}+\frac{1}{8}\right) A_{5,2}+Z_{2,0} A_{3,2}+Z_{3,1} A_{2,1}+Z_{1,1} A_{4,1}+Z_{4,2} A_{1,0}+Z_{2,2} A_{3,0} \text {, } \\
& P_{7,2}=Z_{4,2} A_{3,0}+Z_{3,1} A_{4,1}+Z_{2,0} A_{5,2} \text {, } \\
& P_{2,3}=\left(Z_{0,0}+\frac{1}{8}\right) A_{2,3}+Z_{1,1} A_{1,2}+Z_{2,2} A_{0,1} \text {, } \\
& P_{4,3}=\left(Z_{0,0}+\frac{1}{8}\right) A_{4,3}+Z_{2,0} A_{2,3}+Z_{3,1} A_{1,2}+Z_{1,1} A_{3,2}+Z_{4,2} A_{0,1}+Z_{2,2} A_{2,1}+Z_{3,3} A_{1,0}, \\
& P_{6,3}=\left(Z_{0,0}+\frac{1}{8}\right) A_{6,3}+Z_{2,0} A_{4,3}+Z_{3,1} A_{3,2}+Z_{1,1} A_{5,2}+Z_{4,2} A_{2,1}+Z_{2,2} A_{4,1}+Z_{5,3} A_{1,0}+Z_{3,3} A_{3,0} \\
& P_{8,3}=Z_{5,3} A_{3,0}+Z_{4,2} A_{4,1}+Z_{3,1} A_{5,2}+Z_{2,0} A_{6,3} \text {, } \\
& P_{3,4}=\left(Z_{0,0}+\frac{1}{8}\right) A_{3,4}+Z_{1,1} A_{2,3}+Z_{2,2} A_{1,2}+Z_{3,3} A_{0,1} \text {, } \\
& P_{5,4}=\left(Z_{0,0}+\frac{1}{8}\right) A_{5,4}+Z_{2,0} A_{3,4}+Z_{3,1} A_{2,3}+Z_{1,1} A_{4,3}+Z_{4,2} A_{1,2}+Z_{2,2} A_{3,2}+Z_{5,3} A_{0,1}+Z_{3,3} A_{2,1} \text {, } \\
& P_{7,4}=Z_{5,3} A_{2,1}+Z_{4,2} A_{3,2}+Z_{3,3} A_{4,1}+Z_{3,1} A_{4,3}+Z_{2,2} A_{5,2}+Z_{2,0} A_{5,4}+Z_{1,1} A_{6,3} \text {, } \\
& P_{9,4}=Z_{5,3} A_{4,1}+Z_{4,2} A_{5,2}+Z_{3,1} A_{6,3} \text {, } \\
& P_{4,5}=Z_{3,3} A_{1,2}+Z_{2,2} A_{2,3}+Z_{1,1} A_{3,4}, \\
& P_{6,5}=Z_{5,3} A_{1,2}+Z_{4,2} A_{2,3}+Z_{3,3} A_{3,2}+Z_{3,1} A_{3,4}+Z_{2,2} A_{4,3}+Z_{1,1} A_{5,4} \text {, } \\
& P_{8,5}=Z_{5,3} A_{3,2}+Z_{4,2} A_{4,3}+Z_{3,3} A_{5,2}+Z_{3,1} A_{5,4}+Z_{2,2} A_{6,3} \text {, } \\
& P_{10,5}=Z_{5,3} A_{5,2}+Z_{4,2} A_{6,3}, P_{5,6}=Z_{3,3} A_{2,3}+Z_{2,2} A_{3,4} \text {, } \\
& P_{7,6}=Z_{5,3} A_{2,3}+Z_{4,2} A_{3,4}+Z_{3,3} A_{4,3}+Z_{2,2} A_{5,4} \text {, } \\
& P_{9,6}=Z_{5,3} A_{4,3}+Z_{4,2} A_{5,4}+Z_{3,3} A_{6,3}, P_{11,6}=Z_{5,3} A_{6,3}, P_{6,7}=Z_{3,3} A_{3,4} \text {, } \\
& P_{8,7}=Z_{5,3} A_{3,4}+Z_{3,3} A_{5,4}, P_{10,7}=Z_{5,3} A_{5,4} \text {. }
\end{aligned}
$$

\subsection{Experimental Solution of an Algebraic KdV Problem}

Consider an experimental solution for algebraic order $M=4$, which is a smallest order required to avoid degeneration of the subsequent computational algorithm. Substitution of temporal derivative (7), product (11) of the two-wave solution and the first spatial derivative, third spatial derivative (9), and collection of structural coefficients reduce differential KdV Equation (1) to an experimental algebraic KdV equation 


$$
\begin{aligned}
& \left(B_{1,0}+6 P_{1,0}+T_{1,0}\right) t a+\left(B_{3,0}+6 P_{3,0}+T_{3,0}\right) t a^{3}+\left(B_{5,0}+6 P_{5,0}\right) t a^{5}+\left(B_{0,1}+6 P_{0,1}+T_{0,1}+\left(B_{2,1}+6 P_{2,1}+T_{2,1}\right) t a^{2}\right. \\
& \left.+\left(B_{4,1}+6 P_{4,1}+T_{4,1}\right) t a^{4}+\left(B_{6,1}+6 P_{6,1}\right) t a^{6}\right) T b+\left(\left(B_{1,2}+6 P_{1,2}+T_{1,2}\right) t a+\left(B_{3,2}+6 P_{3,2}+T_{3,2}\right) t a^{3}\right. \\
& \left.+\left(B_{5,2}+6 P_{5,2}+T_{5,2}\right) t a^{5}+\left(B_{7,2}+6 P_{7,2}\right) t a^{7}\right) T b^{2}+\left(B_{0,3}+\left(B_{2,3}+6 P_{2,3}+T_{2,3}\right) t a^{2}+\left(B_{4,3}+6 P_{4,3}+T_{4,3}\right) t a^{4}\right. \\
& \left.+\left(B_{6,3}+6 P_{6,3}+T_{6,3}\right) t a^{6}+\left(B_{8,3}+6 P_{8,3}\right) t a^{8}\right) T b^{3}+\left(B_{1,4} t a+\left(B_{3,4}+6 P_{3,4}+T_{3,4}\right) t a^{3}+\left(B_{5,4}+6 P_{5,4}+T_{5,4}\right) t a^{5}\right. \\
& \left.+\left(B_{7,4}+6 P_{7,4}+T_{7,4}\right) t a^{7}+\left(B_{9,4}+6 P_{9,4}\right) t a^{9}\right) T b^{4}+\left(B_{2,5} t a^{2}+\left(B_{4,5}+6 P_{4,5}+T_{4,5}\right) t a^{4}+\left(B_{6,5}+6 P_{6,5}+T_{6,5}\right) t a^{6}\right. \\
& \left.+\left(B_{8,5}+6 P_{8,5}\right) t a^{8}+6 t a^{10} P_{10,5}\right) T b^{5}+\left(B_{3,6} t a^{3}+\left(B_{5,6}+6 P_{5,6}\right) t a^{5}+\left(B_{7,6}+6 P_{7,6}\right) t a^{7}+6 P_{9,6} t a^{9}+6 P_{11,6} t a^{11}\right) T b^{6} \\
& +\left(B_{4,7} t a^{4}+\left(B_{6,7}+6 P_{6,7}\right) t a^{6}+6 P_{8,7} t a^{8}+6 P_{10,7} t a^{10}+6 P_{12,7} t a^{12}\right) T b^{7}+\left(6 P_{7,8} t a^{7}+6 P_{9,8} t a^{9}+6 P_{11,8} t a^{11}\right. \\
& \left.+6 P_{13,8} t a^{13}\right) T b^{8}+\left(6 P_{8,9} t a^{8}+6 P_{10,9} t a^{10}+6 P_{12,9} t a^{12}\right) T b^{9}=0 .
\end{aligned}
$$

To vanish structural coefficients of $t a^{n} T b^{m}$, construct binomial systems by vanishing structural coefficients of $t a^{k-m} T b^{m}$ of odd binomial orders $k=1,3, \cdots, 2 M-1$ with $m=0,1, \cdots, k$. The binomial systems have two equations, four equations, and five equations for $k=1, k=3$, and $k \geq 5$, respectively.

The degenerated binomial systems for $k=1$ and $k=3$ are, respectively,

$$
\begin{gathered}
T_{1,0}+6 P_{1,0}+B_{1,0}=0, T_{0,1}+6 P_{0,1}+B_{0,1}=0, \\
T_{3,0}+6 P_{3,0}+B_{3,0}=0, \quad T_{2,1}+6 P_{2,1}+B_{2,1}=0, \quad T_{1,2}+6 P_{1,2}+B_{1,2}=0, B_{0,3}=0 .
\end{gathered}
$$

In agreement with one-wave solution (3), structural coefficients $Z_{n, m}$ of the two-wave solution are initialized by

$$
Z_{0,0}=1, \quad Z_{2,0}=-p^{2}
$$

For the experimental solution,

$$
Z_{0,0}=1, \quad Z_{2,0}=-8 / 9 .
$$

Solving Equations (14) and (15) with respect to $Z_{1,1}, Z_{3,1}, Z_{2,2}, Z_{4,2}$, and $Z_{3,3}$ yields

$$
Z_{1,1}=2, Z_{3,1}=-16 / 9, Z_{2,2}=2, Z_{4,2}=-8 / 3, Z_{3,3}=2 \text {. }
$$

For $k=5,7, \cdots, 2 M-3$, the binomial system has five equations

$$
6 P_{5,0}+B_{5,0}=0, \quad T_{4,1}+6 P_{4,1}+B_{4,1}=0, \quad T_{3,2}+6 P_{3,2}+B_{3,2}=0, \quad T_{2,3}+6 P_{2,3}+B_{2,3}=0, \quad B_{1,4}=0 .
$$

Solving first and fifth equations of (19) with respect to $Z_{(k+5) / 2,(k+1) / 2}$ and $Z_{(k+3) / 2,(k+3) / 2}$, respectively, gives

$$
Z_{5,3}=-32 / 9, \quad Z_{4,4}=2 \text {. }
$$

Substitution of (20) in second, third, and fourth equations of system (19) reduces them to identities.

For $k=2 M-1$, the binomial system also has five equations

$$
6 P_{6,1}+B_{6,1}=0, \quad T_{5,2}+6 P_{5,2}+B_{5,2}=0, T_{4,3}+6 P_{4,3}+B_{4,3}=0, \quad T_{3,4}+6 P_{3,4}+B_{3,4}=0, \quad B_{2,5}=0 .
$$

Solving first equation of system (21) respect to $Z_{(k+5) / 2,(k+1) / 2}$ returns

$$
Z_{6,4}=-40 / 9 \text {. }
$$

Substitution of computed structural coefficients (17), (18), (20), and (22) in (4) yields an experimental twowave solution in the ZHT structure of algebraic order $M=4$

$$
\phi(x, t)=\frac{9}{8}-\frac{8}{9} t a^{2}+\left(2 t a-\frac{16}{9} t a^{3}\right) T b+\left(2 t a^{2}-\frac{8}{3} t a^{4}\right) T b^{2}+\left(2 t a^{3}-\frac{32}{9} t a^{5}\right) T b^{3}+\left(2 t a^{4}-\frac{40}{9} t a^{6}\right) T b^{4}
$$

Substitution of the computed structural coefficients in the left-hand-side part of (13) returns an experimental remainder of the ZHT structural approximation 


$$
\begin{aligned}
r_{e}(x, t)= & \left(-\frac{5}{72} t a^{5}+\frac{5}{27} t a^{7}\right) T b^{2}+\left(-\frac{25}{8} t a^{4}+\frac{1055}{72} t a^{6}-\frac{910}{81} t a^{8}\right) T b^{3} \\
& +\left(-\frac{225}{8} t a^{3}+\frac{1765}{8} t a^{5}-\frac{13405}{36} t a^{7}+\frac{14230}{81} t a^{9}\right) T b^{4} \\
& +\left(-\frac{405}{8} t a^{2}+\frac{5805}{8} t a^{4}-\frac{4307}{2} t a^{6}+\frac{19081}{9} t a^{8}-\frac{17536}{27} t a^{10}\right) T b^{5} \\
& +\left(\frac{1215}{8} t a^{3}-\frac{1611}{2} t a^{5}+319 t a^{7}+\frac{3416}{3} t a^{9}-\frac{61760}{81} t a^{11}\right) T b^{6} \\
& +\left(-\frac{405}{4} t a^{4}+189 t a^{6}-190 t a^{8}+904 t a^{10}-\frac{60160}{81} t a^{12}\right) T b^{7} \\
& +\left(-54 t a^{7}+32 t a^{9}+\frac{3920}{9} t a^{11}-\frac{1600}{3} t a^{13}\right) T b^{8} \\
& +\left(-\frac{3200}{9} t a^{12}+320 t a^{10}-72 t a^{8}\right) T b^{9} .
\end{aligned}
$$

Rate of convergence of the ZHT structural approximation is examined in Table 1, where a tolerance

$$
\varepsilon=\max _{t \in(-\infty, \infty) x(-\infty, \infty)}\left|r_{e}(x, t)\right|
$$

and a CPU time $\tau$ are given versus algebraic order $M$ for various reference levels $h$. Table 1 was computed on a workstation in Maple 17.02 by using a six-core AMD-6300 processor with frequency $3.50 \mathrm{GHz}$ and RAM 12.0 GB. The CPU time depends mainly on order of approximation $M$. Tolerance significantly depends upon $M$ and $h$ through interaction parameter $q$. For propagation celerities $U=3$ and $V=1, q=1 / 3, \sqrt{3} / 3, \sqrt{105} / 15=0.333,0.577,0.683$ for $h=1 / 8,0,-1 / 8=0.125,0,-0.125$, respectively. Surface plots of $R_{c}(x, t)$ show a uniform convergence of the two-wave solution in the ZHT structure.

The experimental solutions of Section 2 were computed by experimental programming with lists of equations and expressions in the virtual environment of a global variable Eqe with 4 procedures of 185 code lines in total.

\section{Theory of Interaction of Two Pulsatory Waves in the ZHT Structure}

\begin{tabular}{|c|c|c|c|c|c|c|}
\hline$M$ & & 10 & 20 & 40 & 80 & 160 \\
\hline \multirow[t]{2}{*}{$h=0.125$} & $\varepsilon$ & $1.8 \times 10^{-4}$ & $6.3 \times 10^{-9}$ & $3.7 \times 10^{-18}$ & $6.2 \times 10^{-37}$ & $8.3 \times 10^{-75}$ \\
\hline & $\tau(s)$ & 0.156 & 0.407 & 1.782 & 11.672 & 99.343 \\
\hline \multirow[t]{2}{*}{$h=0.000$} & $\varepsilon$ & 0.125 & 0.00112 & $4.0 \times 10^{-8}$ & $2.4 \times 10^{-17}$ & $3.8 \times 10^{-36}$ \\
\hline & $\tau(s)$ & 0.312 & 0.609 & 2.250 & 13.312 & 111.718 \\
\hline \multirow[t]{2}{*}{$h=-0.125$} & $\varepsilon$ & 1.03 & 0.056 & $5.7 \times 10^{-5}$ & $2.8 \times 10^{-11}$ & $3.4 \times 10^{-24}$ \\
\hline & $\tau(s)$ & 0.250 & 0.578 & 2.328 & 14.282 & 116.812 \\
\hline
\end{tabular}

\subsection{Formulation of the Theoretical Problem in the ZHT Structure}

Compute theoretically a structural solution of (1) for nonlinear interaction of two pulsatory waves. Construct the structural solution in the ZHT structure of algebraic order $M$ and differential order $N$

$$
s(x, t)=h+S \sum_{m=0}^{M+N} T b^{m} \sum_{K=0}^{N+1} C_{m-N+2 k, m} t a^{m-N+2 k},
$$

where $S$ is a scale, $M \rightarrow \infty, C_{m-N+2 k, m}$ is a structural coefficient, $M$ and $N$ are symbolic limits of sum-

Table 1. Convergence of the experimental solutions in the ZHT structure. 
mation. Other variables and parameters are the same as in (2), but instead of experimental instances of section 2 they receive symbolic values to compute theoretically a general term of the structural solution.

In agreement with (26) and (2), a two-wave solution is constructed with algebraic order $M$ and differential order $N=0$ using a generalized Einstein notation for summation, which is extended for exponents, as follows:

$$
\phi(x, t)=h+2 \mu^{2} p^{2}\left(t a^{m} Z_{m, m}+t a^{m+2} Z_{m+2, m}\right) T b^{m},
$$

where $m=0,1, \cdots, M$. When $p=1(q=0), T b=q t b=0$, and initialization condition (16) is invoked, the two- wave solution is reduced to one-wave solution (3).

\subsection{Theoretical Differentiation of the Invariant ZHT Structure}

Primarily, computation a first spatial derivative of a binomial term $\operatorname{ta}^{n} \mathrm{~Tb}^{m}$ yields

$$
\begin{gathered}
\frac{\partial t a^{n} T b^{m}}{\partial x}=\mu\left[d a_{n, m-1} t a^{n} T b^{m-1}+\left(d a_{n-1, m} t a^{n-1}+d a_{n+1, m} t a^{n+1}\right) T b^{m}+d a_{n, m+1} t a^{n} T b^{m+1}\right], \\
d a_{n, m-1}=d a_{n, m-1}(n, m)=q^{2} m, \quad d a_{n-1, m}=d a_{n-1, m}(n, m)=n, \\
d a_{n+1, m}=d a_{n+1, m}(n, m)=-n, \quad d a_{n, m+1}=d a_{n, m+1}(n, m)=-m,
\end{gathered}
$$

where structural coefficients (29) of binomial derivative (28), called differential binomial coefficients, are matrix functions $d a_{n, m}=d a_{n, m}(i, j)$. Here, indices $n$ and $m$, which are equal to powers of ta and $T b$ in the binomial derivative, define names of the matrix functions. Indices $i$ and $j$ which are equal to powers of ta and $T b$ in the binomial term $t a^{i} T b^{j}$, determine definitions of the matrix functions.

Similarly, computation of a first temporal derivative of the binomial term $t a^{n} \mathrm{~Tb}^{m}$ gives

$$
\begin{aligned}
& \frac{\partial t a^{n} T b^{m}}{\partial t}=\mu\left[d t_{n, m-1} t a^{n} T b^{m-1}+\left(d t_{n-1, m} t a^{n-1}+d t_{n+1, m} t a^{n+1}\right) T b^{m}+d t_{n, m+1} t a^{n} T b^{m+1}\right], \\
& d t_{n, m-1}=d t_{n, m-1}(n, m)=-2 q^{2}\left(2 \mu^{2} q^{2}+3 h\right) m, \quad d t_{n-1, m}=d t_{n-1, m}(n, m)=-2\left(2 \mu^{2}+3 h\right) n, \\
& d t_{n+1, m}=d t_{n+1, m}(n, m)=2\left(2 \mu^{2}+3 h\right) n, \quad d t_{n, m+1}=d t_{n, m+1}(n, m)=2\left(2 \mu^{2} q^{2}+3 h\right) m .
\end{aligned}
$$

Thus, spatial and temporal derivatives (28) and (30) have the same structure but vary in differential binomial coefficients (29) and (31), respectively.

Increase in order of differentiation produces a two-dimensional (2d) differentiation cascade, which is shown in Figure 1. The one-dimensional (1d) differentiation cascade of invariant hyperbolic-secant structures is asymmetrical as the cascade spreads only towards higher powers [18]. To the contrary of the $1 \mathrm{~d}$ differentiation cascade, the $2 \mathrm{~d}$ differentiation cascade spreads in symmetrical square waves, which resemble circular waves on the water surface generated by a point source. Similar to the $1 \mathrm{~d}$ differentiation of the invariant trigonometric, hyperbolic, and elliptic structures [18], the 2d differentiation of an even order preserves structure of binomial derivatives and the $2 \mathrm{~d}$ differentiation of an odd order converts structure of binomial derivatives to complementary ones.

Finally, computation a third spatial derivative of the binomial term $t a^{n} T b^{m}$ returns a binomial derivative of the following structure:

$$
\begin{aligned}
\frac{\partial^{3} t a^{n} T b^{m}}{\partial x^{3}}= & \mu^{3}\left[d b_{n, m-3} t a^{n} T b^{m-3}+\left(d b_{n-1, m-2} t a^{n-1}+d b_{n+1, m-2} t a^{n+1}\right) T b^{m-2}+\left(d b_{n-2, m-1} t a^{n-2}+d b_{n, m-1} t a^{n}\right.\right. \\
& \left.+d b_{n+2, m-1} t a^{n+2}\right) T b^{m-1}+\left(d b_{n-3, m} t a^{n-3}+d b_{n-1, m} t a^{n-1}+d b_{n+1, m} t a^{n+1}+d b_{n+3, m} t a^{n+3}\right) T b^{m} \\
& +\left(d b_{n-2, m+1} t a^{n-2}+d b_{n, m+1} t a^{n}+d b_{n+2, m+1} t a^{n+2}\right) T b^{m+1} \\
& +\left(d b_{n-1, m+2} t a^{n-1}+d b_{n+1, m+2} t a^{n+1}\right) T b^{m+2} \\
& \left.+d b_{n, m+3} t a^{n} T b^{m+3}\right]
\end{aligned}
$$

with differential binomial coefficients 


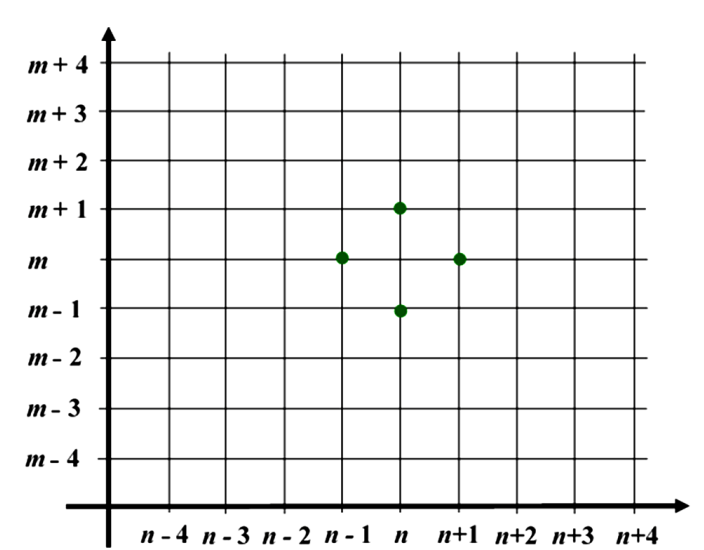

(a)

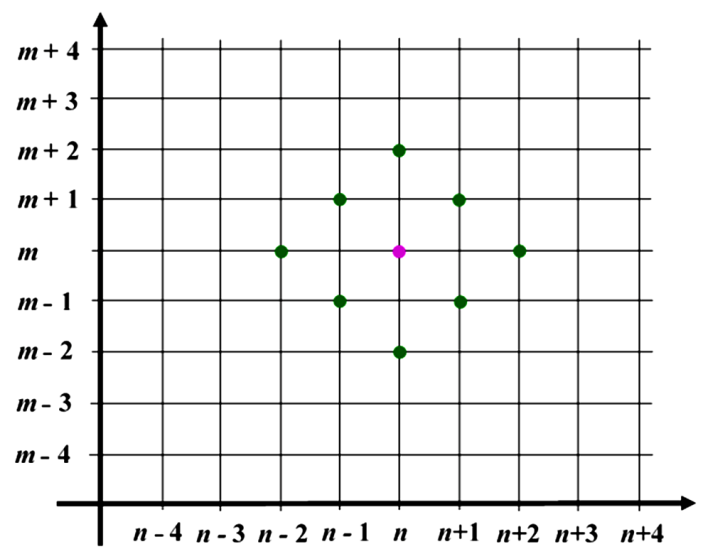

(c)

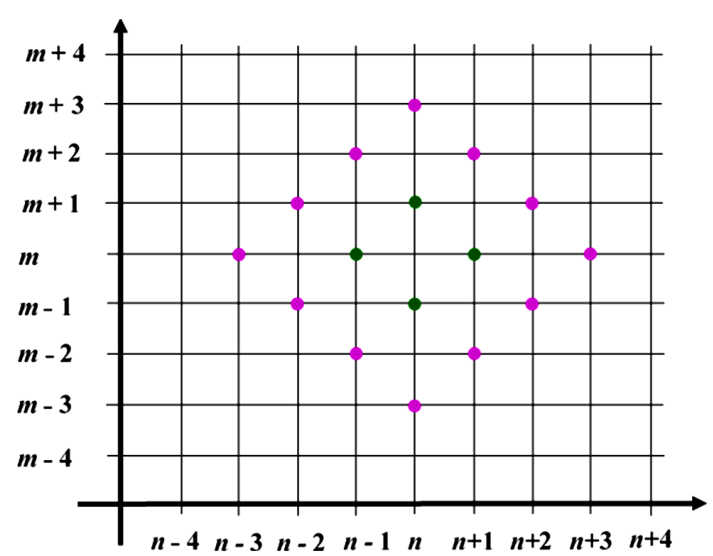

(b)

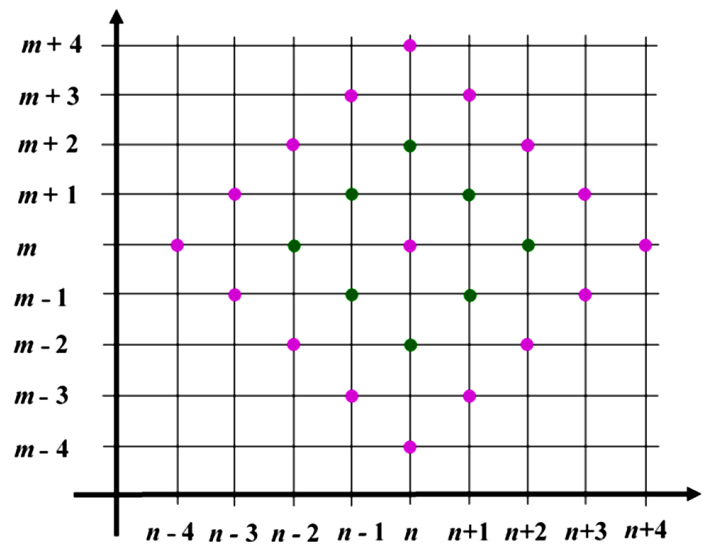

(d)

Figure 1. Differentiation cascade of the binomial structure: (a) $N=1$; (b) $N=3$; (c) $N=2$; (d) $N=4$.

$$
\begin{aligned}
& d b_{n, m-3}=d b_{n, m-3}(n, m)=q^{6} m(m-1)(m-2), d b_{n-1, m-2}=d b_{n-1, m-2}(n, m)=3 q^{4} m(m-1) n, \\
& d b_{n+1, m-2}=d b_{n+1, m-2}(n, m)=-3 q^{4} m(m-1) n, d b_{n-2, m-1}=d b_{n-2, m-1}(n, m)=3 q^{2} m n(n-1), \\
& d b_{n, m-1}=d b_{n, m-1}(n, m)=-q^{2} m\left[2 q^{2}+3 q^{2} m(m-1)+6 n^{2}\right], d b_{n+2, m-1}=d b_{n+2, m-1}(n, m)=3 q^{2} m n(n+1), \\
& d b_{n-3, m}=d b_{n-3, m}(n, m)=n(n-1)(n-2), d b_{n-1, m}=d b_{n-1, m}(n, m)=-\left[2+6 q^{2} m^{2}+3 n(n-1)\right] n, \\
& d b_{n+1, m}=d b_{n+1, m}(n, m)=\left[2+6 q^{2} m^{2}+3 n(n+1)\right] n, d b_{n+3, m}=d b_{n+3, m}(n, m)=-n(n+1)(n+2), \\
& d b_{n-2, m+1}=d b_{n-2, m+1}(n, m)=-3 m n(n-1), d b_{n, m+1}=d b_{n, m+1}(n, m)=m\left[2 q^{2}+3 q^{2} m(m+1)+6 n^{2}\right], \\
& d b_{n+2, m+1}=d b_{n+2, m+1}(n, m)=-3 m n(n+1), d b_{n-1, m+2}=d b_{n-1, m+2}(n, m)=3 m(m+1) n, \\
& d b_{n+1, m+2}=d b_{n+1, m+2}(n, m)=-3 m(m+1) n, d b_{n, m+3}=d b_{n, m+3}(n, m)=-m(m+1)(m+2) .
\end{aligned}
$$

Construct now a sequence of terms of the zigzag structure $\left(Z_{m+k, m} t a^{m+k}+Z_{m+k+2, m} t a^{m+k+2}\right) T b^{m+k}$ with $k=-N,-N+1, \cdots, N$, derivatives of which make a contribution to the general term of a zigzag derivative through binomial derivatives (28), (30), and (32). Substitute then the binomial derivatives of $t a^{m+k} T b^{m+k}, t a^{m+k+2} T b^{m+k}$ and collect like terms of a structural coefficient of the general term proportional to $T b^{m}$. In agreement with (5), a first spatial derivative of the two-wave solution is the invariant ZHT structure of algebraic order $M+N$ and differential order $N=1$

$$
\frac{\partial \phi}{\partial x}=2 \mu^{3} p^{2}\left(A_{m-1, m} t a^{m-1}+A_{m+1, m} t a^{m+1}+A_{m+3, m} t a^{m+3}\right) T b^{m},
$$


where $m=0,1, \cdots, M+N$ and structural coefficients $A_{n, m}$ are following:

$$
\begin{aligned}
& A_{m-1, m}=D a_{n, m+1} Z_{m-1, m-1}+D a_{n-1, m} Z_{m, m}, \\
& A_{m+1, m}=D a_{n, m+1} Z_{m+1, m-1}+D a_{n+1, m} Z_{m, m}+D a_{n-1, m} Z_{m+2, m}+D a_{n, m-1} Z_{m+1, m+1}, \\
& A_{m+3, m}=D a_{n+1, m} Z_{m+2, m}+D a_{n, m-1} Z_{m+3, m+1}, \\
& D a_{n, m} Z_{i, j}=d a_{n, m}(i, j) Z_{i, j} .
\end{aligned}
$$

Equations (34)-(35) are complemented by truncation conditions of the two-wave solution

$$
Z_{n, m}=0 \text { for } m<0, m>M \text {, }
$$

which are set by (27). Conditions (36) result in truncation conditions for the first spatial derivative:

$$
A_{n, m}=0 \text { for } n<0, n<m-1, n>m+3 ; A_{m+3, m}=0 \text { for } m>M ; A_{m+1, m}=0, A_{m-1, m}=0 \text { for } m>M+1 \text {. }
$$

Similarly, a first temporal derivative of the two wave solution again yields the invariant ZHT structure of algebraic order $M+N$ and differential order $N=1$

$$
\frac{\partial \phi}{\partial t}=2 \mu^{3} p^{2}\left(T_{m-1, m} t a^{m-1}+T_{m+1, m} t a^{m+1}+T_{m+3, m} t a^{m+3}\right) T b^{m},
$$

where structural coefficients $T_{n, m}$ are

$$
\begin{aligned}
& T_{m-1, m}=D t_{n, m+1} Z_{m-1, m-1}+D t_{n-1, m} Z_{m, m}, \quad T_{m+1, m}=D t_{n, m+1} Z_{m+1, m-1}+D t_{n+1, m} Z_{m, m}+D t_{n-1, m} Z_{m+2, m} \\
& +D t_{n, m-1} Z_{m+1, m+1}, \quad T_{m+3, m}=D t_{n+1, m} Z_{m+2, m}+D t_{n, m-1} Z_{m+3, m+1}, \quad D t_{n, m} Z_{i, j}=d t_{n, m}(i, j) Z_{i, j},
\end{aligned}
$$

and truncation conditions are given by (36).Truncation conditions for the first temporal derivative become

$$
T_{n, m}=0 \text { for } n<0, n<m-1, n>m+3 ; T_{m+3, m}=0 \text { for } m>M ; T_{m+1, m}=0, T_{m-1, m}=0 \text { for } m>M+1 .
$$

Finally, computation of a third spatial derivative of the two-wave solution produces the invariant ZHT structure of algebraic order $M+N$ and differential order $N=3$

$$
\frac{\partial^{3} \phi}{\partial x^{3}}=2 \mu^{5} p^{2}\left(B_{m-3, m} t a^{m-3}+B_{m-1, m} t a^{m-1}+B_{m+1, m} t a^{m+1}+B_{m+3, m} t a^{m+3}+B_{m+5, m} t a^{m+5}\right) T b^{m},
$$

where structural coefficients $B_{n, m}$ are

$$
\begin{aligned}
B_{m-3, m}= & D b_{n, m+3} Z_{m-3, m-3}+D b_{n-1, m+2} Z_{m-2, m-2}+D b_{n-2, m+1} Z_{m-1, m-1}+D b_{n-3, m} Z_{m, m}, \\
B_{m-1, m}= & D b_{n, m+3} Z_{m-1, m-3}+D b_{n+1, m+2} Z_{m-2, m-2}+D b_{n-1, m+2} Z_{m, m-2}+D b_{n, m+1} Z_{m-1, m-1} \\
& +D b_{n-2, m+1} Z_{m+1, m-1}+D b_{n-1, m} Z_{m, m}+D b_{n-3, m} Z_{m+2, m}+D b_{n-2, m-1} Z_{m+1, m+1}, \\
B_{m+1, m}= & D b_{n+1, m+2} Z_{m, m-2}+D b_{n+2, m+1} Z_{m-1, m-1}+D b_{n, m+1} Z_{m+1, m-1}+D b_{n+1, m} Z_{m, m} \\
& +D b_{n-1, m} Z_{m+2, m}+D b_{n, m-1} Z_{m+1, m+1}+D b_{n-2, m-1} Z_{m+3, m+1}+D b_{n-1, m-2} Z_{m+2, m+2}, \\
B_{m+3, m}= & D b_{n+2, m+1} Z_{m+1, m-1}+D b_{n+3, m} Z_{m, m}+D b_{n+1, m} Z_{m+2, m}+D b_{n+2, m-1} Z_{m+1, m+1} \\
& +D b_{n, m-1} Z_{m+3, m+1}+D b_{n+1, m-2} Z_{m+2, m+2}+D b_{n-1, m-2} Z_{m+4, m+2}+D b_{n, m-3} Z_{m+3, m+3}, \\
B_{m+5, m}= & D b_{n+3, m} Z_{m+2, m}+D b_{n+2, m-1} Z_{m+3, m+1}+D b_{n+1, m-2} Z_{m+4, m+2}+D b_{n, m-3} Z_{m+5, m+3}, \\
D b_{n, m} Z_{i, j} & =d b_{n, m}(i, j) Z_{i, j},
\end{aligned}
$$

and truncation conditions are set by (36). Truncation conditions for the third spatial derivative are

$$
\begin{aligned}
& B_{n, m}=0 \text { for } n<0, n<m-3, n>m+5 ; B_{m+5, m}=0 \text { for } m>M ; B_{m+3, m}=0 \text { for } m>M+1 ; \\
& B_{m+1, m}=0 \text { for } m>M+2 ; B_{m-1, m}=0, B_{m-3, m}=0 \text { for } m>M+3 .
\end{aligned}
$$

Equations (34), (38), and (41) show that the ZHT structure is invariant with respect to differentiation of orders $N=1$ and $N=3$, which only modify algebraic orders and structural coefficients. The zigzag structure of twowave solution (27) and its derivatives (34) and (38) together with product of (27) and (34) are shown in Figure 2 in a virtual space of computational indices $n$ and $m$ of structural coefficients $A_{n, m}, T_{n, m}, B_{n, m}$ 


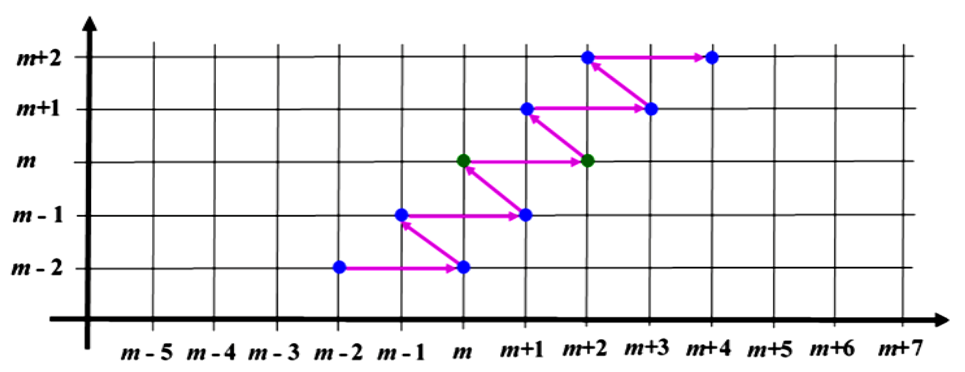

(a)

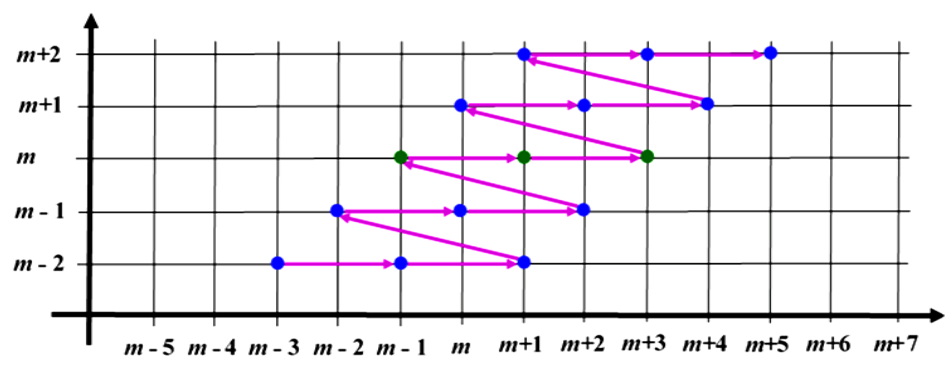

(b)

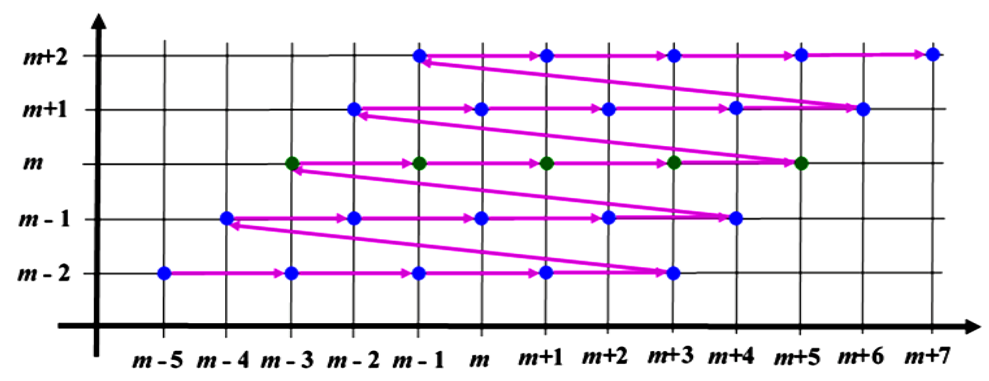

(c)

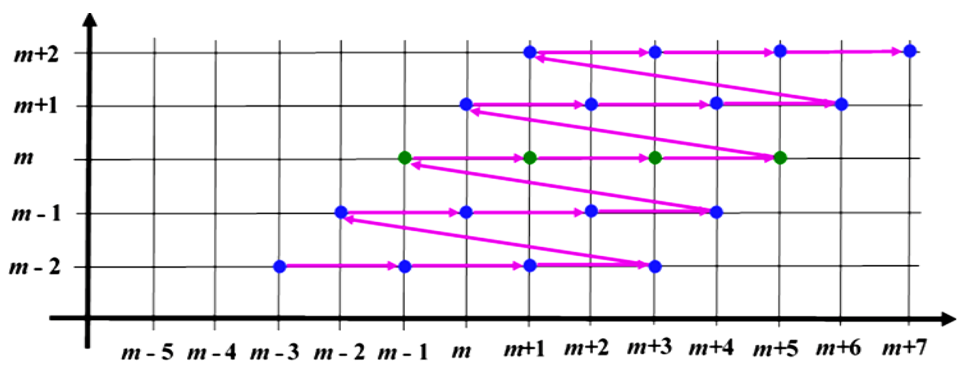

(d)

Figure 2. The invariant ZHT structure in the virtual space $(n, m)$ : (a)-(27), (b)-(34), (c)-(38), (d)-(53).

and $P_{n, m}$, which coincides with a virtual space of computational powers $n$ and $m$ of $t a^{n} T b^{m}$. Differentiation increases the width of the invariant ZHT structure and the effect of multiplication is similar to that of differentiation.

\subsection{Theoretical Multiplication of the Invariant ZHT Structures}

Continuation of spatial derivatives (34) and (38) in the invariant ZHT structure to any differential order $N$ in the generalized Einstein notation gives

$$
\frac{\partial^{N} \phi}{\partial x^{N}}=2 \mu^{N+2} p^{2} R_{m-N+2 k, m} t a^{m-N+2 k} T b^{m}
$$


where $k=0,1, \cdots, N+1$ and $m=0,1, \cdots, M+N$. The spatial derivative of order $N-1$ then becomes

$$
\frac{\partial^{N-1} \phi}{\partial x^{N-1}}=2 \mu^{N+1} p^{2} Q_{m-N+2 k+1, m} t a^{m-N+2 k+1} T b^{m} .
$$

Differentiation of (45) with respect to $x$ and reduction of all terms to a general term by substitutions $m=m+1$ and $k=k-1$ in a term proportional to $T b^{m-1}, k=k-1$ in a term proportional to $t a^{m-N+2 k+2} T b^{m}, m=m-1$ in a term proportional to $T b^{m+1}$ yields

$$
\begin{aligned}
\frac{\partial^{N} \phi}{\partial x^{N}}= & 2 \mu^{N+2} p^{2}\left[-(m-N+2 k-1) Q_{m-N+2 k-1, m}-(m-1) Q_{m-N+2 k, m-1}\right. \\
& \left.+(m-N+2 k-1) Q_{m-N+2 k+1, m}+(m+1) q^{2} Q_{m-N+2 k, m+1}\right] t a^{m-N+2 k} T^{m},
\end{aligned}
$$

where structural coefficients of (44) are connected with structural coefficients of (46) by a recurrent relation

$$
\begin{aligned}
R_{m-N+2 k, m}= & -(m-N+2 k-1) Q_{m-N+2 k-1, m}-(m-1) Q_{m-N+2 k, m-1} \\
& +(m-N+2 k+1) Q_{m-N+2 k+1, m}+(m+1) q^{2} Q_{m-N+2 k, m+1} .
\end{aligned}
$$

Thus, the invariance of the ZHT structure with respect to spatial differentiation of any order is proved by induction.

Set up two spatial derivatives of the two-wave solution in the invariant ZHT structures of algebraic order $M$ and differential orders $N_{1}$ and $N_{2}$ with structural coefficients $Q_{m-N_{1}+2 k, m}$ and $R_{m-N_{2}+2 k, m}$ as

$$
\frac{\partial^{N_{1}} \phi}{\partial x^{N_{1}}}=2 \mu^{N_{1}+2} p^{2} Q_{m-N_{1}+2 k, m} t a^{m-N_{1}+2 k} T b^{m}, \quad \frac{\partial^{N_{2}} \phi}{\partial x^{N_{2}}}=2 \mu^{N_{2}+2} p^{2} R_{m-N_{2}+2 k, m} t a^{m-N_{2}+2 k} T b^{m},
$$

where $k=0,1, \cdots, N_{1}+1$ and $m=0,1, \cdots, M+N_{1}$ for the first structure, $k=0,1, \cdots, N_{2}+1$ and $m=0,1, \cdots, M+N_{2}$ for the second structure. Product of (48) with a binomial substitution $m=n, k=l$ and (49) with a binomial substitution $m=m-n, k=k-l$ returns

$$
\frac{\partial^{N_{1}} \phi}{\partial x^{N_{1}}} \frac{\partial^{N_{2}} \phi}{\partial x^{N_{2}}}=4 \mu^{N_{1}+N_{2}+4} p^{4} Q_{n-N_{1}+2 l, n} R_{m-n-N_{2}+2 k-2 l, m-n} t a^{m-N_{1}-N_{2}+2 k} T b^{m},
$$

where $l=0,1, \cdots, k, \quad k=0,1, \cdots N_{1}+N_{2}+2, \quad n=0,1, \cdots, m$ and $m=0,1, \cdots, 2 M+N_{1}+N_{2}$.

Thus, the ZHT structure is also invariant with respect to multiplication since a general term of the product is the ZHT structure of algebraic order $2 M+N_{1}+N_{2}$ and differentiation order $N_{1}+N_{2}$

$$
\frac{\partial^{N_{1}} \phi}{\partial x^{N_{1}}} \frac{\partial^{N_{2}} \phi}{\partial x^{N_{2}}}=4 \mu^{N_{1}+N_{2}+4} p^{4} P_{m-N_{1}-N_{2}+2 k} t a^{m-N_{1}-N_{2}+2 k} T b^{m},
$$

where the structural coefficients are

$$
P_{m-N_{1}-N_{2}+2 k}=Q_{n-N_{1}+2 l, n} R_{m-n-N_{2}+2 k-2 l, m-n} .
$$

Summation of a general term of the product of two-wave solution (27) and first spatial derivative (34) by (51)-(52) with $N_{1}=0, N_{2}=1, k=0,1, \cdots, 3$ yields

$$
\phi \frac{\partial \phi}{\partial x}=4 \mu^{5} p^{4}\left(P_{m-1, m} t a^{m-1}+P_{m+1, m} t a^{m+1}+P_{m+3, m} t a^{m+3}+P_{m+5, m} t a^{m+5}\right) T b^{m},
$$

where structural coefficients $P_{m-1+2 k, m}$ are obtained for $Q=Z$ and $R=A$ by constructing a list of sums of general terms for $l=0,1, \cdots, k$ with truncation conditions $Z_{n+4, n}=0, Z_{n+6, n}=0$, which follows from (27),

$$
\begin{aligned}
& P_{m-1, m}=A_{m-n-1, m-n} Z_{n, n}, P_{m+1, m}=A_{m-n+1, m-n} Z_{n, n}+A_{m-n-1, m-n} Z_{n+2, n}, \\
& P_{m+3, m}=A_{m-n+3, m-n} Z_{n, n}+A_{m-n+1, m-n} Z_{n+2, n}, P_{m+5, m}=A_{m-n+5, m-n} Z_{n, n}+A_{m-n+3, m-n} Z_{n+2, n} .
\end{aligned}
$$

Structure of the two-wave solution is reduced to that of a spatial derivative by substitution

$$
Z_{0,0}=Z_{0,0}+h / 2 \mu^{2} p^{2} .
$$

Truncation conditions for product of the two-wave solution and the first spatial derivative become 


$$
\begin{aligned}
& P_{n, m}=0 \text { for } n<0, n<m-1, n>m+5 ; P_{m+5, m}=0 \text { for } m>2 M ; \\
& P_{m+3, m}=0, P_{m+1, m}=0, P_{m-1, m}=0, P_{m-3, m}=0 \text { for } m>2 M+1
\end{aligned}
$$

\subsection{Theoretical Solution of the Algebraic KdV Problem}

Substitution of temporal derivative (38), product (53) of the two-wave solution and the first order spatial derivative, third order spatial derivative (41), and collection of structural coefficients reduce differential KdV Equation (1) to a theoretical algebraic KdV equation

$$
\begin{aligned}
& {\left[2 \mu^{5} p^{2} B_{m-3, m} t a^{m-3}+\left(2 \mu^{3} p^{2} T_{m-1, m}+24 \mu^{5} p^{4} P_{m-1, m}+2 \mu^{5} p^{2} B_{m-1, m}\right) t a^{m-1}\right.} \\
& +\left(2 \mu^{3} p^{2} T_{m+1, m}+24 \mu^{5} p^{4} P_{m+1, m}+2 \mu^{5} p^{2} B_{m+1, m}\right) t a^{m+1}+\left(2 \mu^{3} p^{2} T_{m+3, m}+24 \mu^{5} p^{4} P_{m+3, m}+2 \mu^{5} p^{2} B_{m+3, m}\right) t a^{m+3} \\
& \left.+\left(24 \mu^{5} p^{4} P_{m+5, m}+2 \mu^{5} p^{2} B_{m+5, m}\right) t a^{m+5}\right] T b^{m}=0
\end{aligned}
$$

which is complemented by truncation conditions (40), (56), and (43).

For this equation to be satisfied exactly for all parameters and variables, all structural coefficients of $t a^{n} T b^{m}$ should vanish. Therefore, five structural coefficients of (57), which are supposed to be vanished, are

$$
\begin{aligned}
& \mu^{2} B_{m-3, m}=0, T_{m-1, m}+12 \mu^{2} p^{2} P_{m-1, m}+\mu^{2} B_{m-1, m}=0, T_{m+1, m}+12 \mu^{2} p^{2} P_{m+1, m}+\mu^{2} B_{m+1, m}=0, \\
& T_{m+3, m}+12 \mu^{2} p^{2} P_{m+3, m}+\mu^{2} B_{m+3, m}=0,12 \mu^{2} p^{2} P_{m+5, m}+\mu^{2} B_{m+5, m}=0 .
\end{aligned}
$$

These equations constitute a polynomial system of equations with respect to leading structural coefficients $Z_{n, m}$ of increasing orders. To combine equations with respect to $Z_{n, m}$ of same binomial orders $k$, construct binomial systems as structural coefficients of $t a^{k-m} T b^{m}$, where $k=1,3, \cdots 2 M-1$ for $m=0,1, \cdots k$.

The binomial systems of orders $k=1$ and $k=3$ have two and four equations, respectively,

$$
\begin{gathered}
T_{1,0}+12 \mu^{2} p^{2} P_{1,0}+\mu^{2} B_{1,0}=0, T_{0,1}+12 \mu^{2} p^{2} P_{0,1}+\mu^{2} B_{0,1}=0, \\
T_{3,0}+12 \mu^{2} p^{2} P_{3,0}+\mu^{2} B_{3,0}=0, T_{2,1}+12 \mu^{2} p^{2} P_{2,1}+\mu^{2} B_{2,1}=0, T_{1,2}+12 \mu^{2} p^{2} P_{1,2}+\mu^{2} B_{1,2}=0, \mu^{2} B_{0,3}=0 .
\end{gathered}
$$

Substituting structural coefficients $T_{n, m}, P_{n, m}, B_{n, m}$ through $Z_{n, m}$ by (39)-(40), (54)-(56), (42)-(43), using initialization (16), and solving (59)-(60) with respect to $Z_{1,1}, Z_{3,1}, Z_{2,2}, Z_{4,2}$ and $Z_{3,3}$ gives

$$
Z_{1,1}=2, Z_{3,1}=-p^{2}, Z_{2,2}=2, Z_{4,2}=-2 p^{2}, Z_{3,3}=2 .
$$

For binomial orders $k=5,7, \cdots 2 M-3$, where $k=2 l+5$ for $l=0,1, \cdots M-4$, the binomial system has five equations

$$
\begin{aligned}
& 12 \mu^{2} p^{2} P_{l+5, l}+\mu^{2} B_{l+5, l}=0, T_{l+4, l+1}+12 \mu^{2} p^{2} P_{l+4, l+1}+\mu^{2} B_{l+4, l+1}=0, \\
& T_{l+3, l+2}+12 \mu^{2} p^{2} P_{l+3, l+2}+\mu^{2} B_{l+3, l+2}=0, \\
& T_{l+2, l+3}+12 \mu^{2} p^{2} P_{l+2, l+3}+\mu^{2} B_{l+2, l+3}=0, \mu^{2} B_{l+1, l+4}=0 .
\end{aligned}
$$

Solving first and fifth equations of (62) with respect to $Z_{(k+5) / 2,(k+1) / 2}$ and $Z_{(k+3) / 2,(k+3) / 2}$ respectively, yields

$$
Z_{5,3}=-4 p^{2}, Z_{4,4}=2, Z_{6,4}=-5 p^{2}, Z_{5,5}=2, \cdots Z_{M+1, M-1}=-M p^{2}, Z_{M, M}=2,
$$

where last two terms are obtained by induction. In agreement with the experimental solution of section 2.4, second, third, and fourth equations of system (62) are satisfied identically.

For binomial orders $k=7,9, \cdots 2 M-1$, where $k=2 l+5$ for $l=M-3$, the binomial system also has five equations

$$
\begin{aligned}
& 12 \mu^{2} p^{2} P_{M+2, M-3}+\mu^{2} B_{M+2, M-3}=0, T_{M+1, M-2}+12 \mu^{2} p^{2} P_{M+1, M-2}+\mu^{2} B_{M+1, M-2}=0, \\
& T_{M, M-1}+12 \mu^{2} p^{2} P_{M, M-1}+\mu^{2} B_{M, M-1}=0, T_{M-1, M}+12 \mu^{2} p^{2} P_{M-1, M}+\mu^{2} B_{M-1, M}=0, \mu^{2} B_{M-2, M+1}=0 .
\end{aligned}
$$

Solving first equation of (64) with respect to $Z_{(k+5) / 2,(k+1) / 2}$ returns 


$$
Z_{6,4}=-5 p^{2}, Z_{7,5}=-6 p^{2}, \cdots Z_{M+2, M}=-(M+1) p^{2},
$$

where a last term is also generated by induction, the proof of which will be completed in Section 4 . Finally, a general solution for the ZHT structural coefficients may be written as

$$
Z_{m, m}=2, Z_{m+2, m}=-(m+1) p^{2},
$$

and general term (27) of the two-wave solution in the ZHT structure becomes

$$
\phi(x, t)=h+2 \mu^{2} p^{2}\left[2 t a^{m}-(m+1) p^{2} t a^{m+2}\right] T b^{m}
$$

for $m=1,2, \cdots M$.Theoretical formula for the ZHT structural sum of the two-wave solution is

$$
\phi(x, t)=h+2 \mu^{2} p^{2}\left\{1-p^{2} t a^{2}+\sum_{m=1}^{M}\left[2 t a^{m}-(m+1) p^{2} t a^{m+2}\right] T b^{m}\right\} .
$$

Substitution of initialized (16) and computed (66) structural coefficients in the left-hand-side part of theoretical algebraic KdV Equation (57) yields a theoretical remainder of the invariant ZHT structure

$$
\begin{aligned}
r_{t}(x, t)= & 2 \mu^{3} p^{2}\left[\left(C_{M+1, M-2} t a^{M+1}+C_{M+3, M-2} t a^{M+3}\right) T b^{M-2}+\left(C_{M, M-1} t a^{M}+C_{M+2, M-1} t a^{M+2}\right.\right. \\
& \left.+C_{M+4, M-1} t a^{M+4}\right) T b^{M-1}+\left(C_{M-1, M} t a^{M-1}+C_{M+1, M} t a^{M+1}+C_{M+3, M} t a^{M+3}+C_{M+5, M} t a^{M+5}\right) T b^{M} \\
& \left.+\sum_{m=M+1}^{2 M+1}\left(C_{m-3, m} t a^{m-3}+C_{m-1, m} t a^{m-1}+C_{m+1, m} t a^{m+1}+C_{m+3, m} t a^{m+3}+C_{m+5, m} t a^{m+5}\right) T b^{m}\right],
\end{aligned}
$$

where a structural coefficient

$$
C_{n, m}=T_{n, m}+12 \mu^{2} p^{2} P_{n, m}+\mu^{2} B_{n, m}
$$

and truncation conditions (40), (56), and (43) are invoked.

The theoretical solutions of Section 3 in the invariant ZHT structure were computed using theoretical programming methods with symbolic general terms by the generalized Einstein notation in the virtual environment of a global variable Eqt with 16 procedures of 923 code lines in total. The theoretical formulas for two-wave solution (68), first spatial derivative (34)-(36), first temporal derivative (38)-(40), third spatial derivative (41)-(43), product of the two-wave solution and the first spatial derivative (53)-(56), and structural remainder (69)-(70) were justified by the correspondent experimental solutions for algebraic order $M=40$.

\section{Discussion and Visualization}

Through an expansion variable $z=t a T b=q t a t b$, where $|z|<1$ as $-1 \leq t a \leq 1,-1 \leq t b \leq 1$, and $0 \leq q<1$, summation of the ZHT structural approximation yields

$$
\phi(x, t)=h+\frac{2 \mu^{2} p^{2}\left(1-p^{2} t a^{2}-t a^{2} T b^{2}\right)}{(1-t a T b)^{2}},
$$

since a partial sum of the Taylor series expansion of (71) in $Z$ of order $M$ returns the same expression as the ZHT structural sum (68) with the same general term as general term (67) of the invariant ZHT structure.

A functional form of (71) expressed through two regular hyperbolic functions $\tanh (\mu X)$ and $\tanh (v Y)$, five parameters $h, q, p, \mu, v$, and two propagation variables $X=x-U t-a$ and $Y=x-V t-b$ becomes

$$
\phi(x, t)=h+\frac{2 \mu^{2} p^{2}\left[1-p^{2} \tanh ^{2}(\mu X)-q^{2} \tanh ^{2}(\mu X) \tanh ^{2}(v Y)\right]}{[1-q \tanh (\mu X) \tanh (v Y)]^{2}} .
$$

Differentiation and substitution of Equation (72) into differential KdV Equation (1) completes the proof by induction of (66)-(68) and returns another verification of (71) as Equation (1) is satisfied identically.

Conversion of (72) through two singular hyperbolic functions $\operatorname{coth}(\mu X)$ and $\operatorname{csch}(\mu X)$, two regular hyperbolic functions $\tanh (v Y)$ and $\operatorname{sech}(v Y)$, three parameters $h, \mu, v$ and two variables $X$ and $Y$ gives 


$$
\phi(x, t)=h+\frac{2\left(\mu^{2}-v^{2}\right)\left[\mu^{2} \operatorname{csch}^{2}(\mu X)+v^{2} \operatorname{sech}^{2}(v Y)\right]}{[\mu \operatorname{coth}(\mu X)-v \tanh (v Y)]^{2}} .
$$

When $h=0$, the two-wave solution reduces to the conventional form [6] of the two-soliton solution in two singular hyperbolic functions $\operatorname{coth}(\sqrt{U} X / 2)$ and $\operatorname{csch}(\sqrt{U} X / 2)$, two regular hyperbolic functions $\tanh (\sqrt{V} Y / 2)$ and $\operatorname{sech}(\sqrt{V} Y / 2)$, two parameters $U$ and $V$, and two propagation variables $X$ and $Y$

$$
\phi(x, t)=\frac{(U-V)\left[U \operatorname{csch}^{2}(\sqrt{U} X / 2)+V \operatorname{sech}^{2}(\sqrt{V} Y / 2)\right]}{2[\sqrt{U} \operatorname{coth}(\sqrt{U} X / 2)-\sqrt{V} \tanh (\sqrt{V} Y / 2)]^{2}} .
$$

A remainder of the Taylor series approximation of two-wave solution (71)

$$
r_{s}(x, t)=2 \mu^{2} p^{2} \sum_{m=M+1}^{\infty}\left[2-(m+1) p^{2} t a^{2}\right] t a^{m} T b^{m}
$$

converges slower than the ZHT structural remainder (69) because of the infinite limit of summation and truncation conditions (40), (56), and (43). So, the method of decomposition in the invariant ZHT structure is more robust than the method of expansion in the Taylor series.

Interaction of two pulsatory waves is visualized by spatiotemporal plots in Figure 3 for positive and negative reference levels $h$. Negative values of $h$ considerably increase amplitudes and decrease dispersions of pulsatory waves compared with those of solitons with $h=0$, because pulsatory waves propagate on a more shallow water in this case. The effect of positive values of $h$ is opposite and results in decrease of amplitudes and increase of dispersions. Similar to interaction of two solitons, interaction of two pulsatory waves is also conservative and preserves one-wave solutions before and after a nonlinear interaction at the moment of merging. Animations of the two-wave solution show that the merging process may be considered as a flow of a faster fluid of the first pulsatory wave into the second pulsatory wave with a slower fluid.

\section{Conclusions}

The analytical methods of solving PDEs by undetermined coefficients and series expansions are generalized by the computational method of solving nonlinear PDEs by decomposition in the invariant ZHT structure. The computational algorithm is developed by experimental computing using lists of equations and expressions implemented in four procedures of 185 code lines in total. Afterwards, the computational method is proved by theoretical computing with symbolic general terms implemented in 16 procedures with 923 code lines in total.
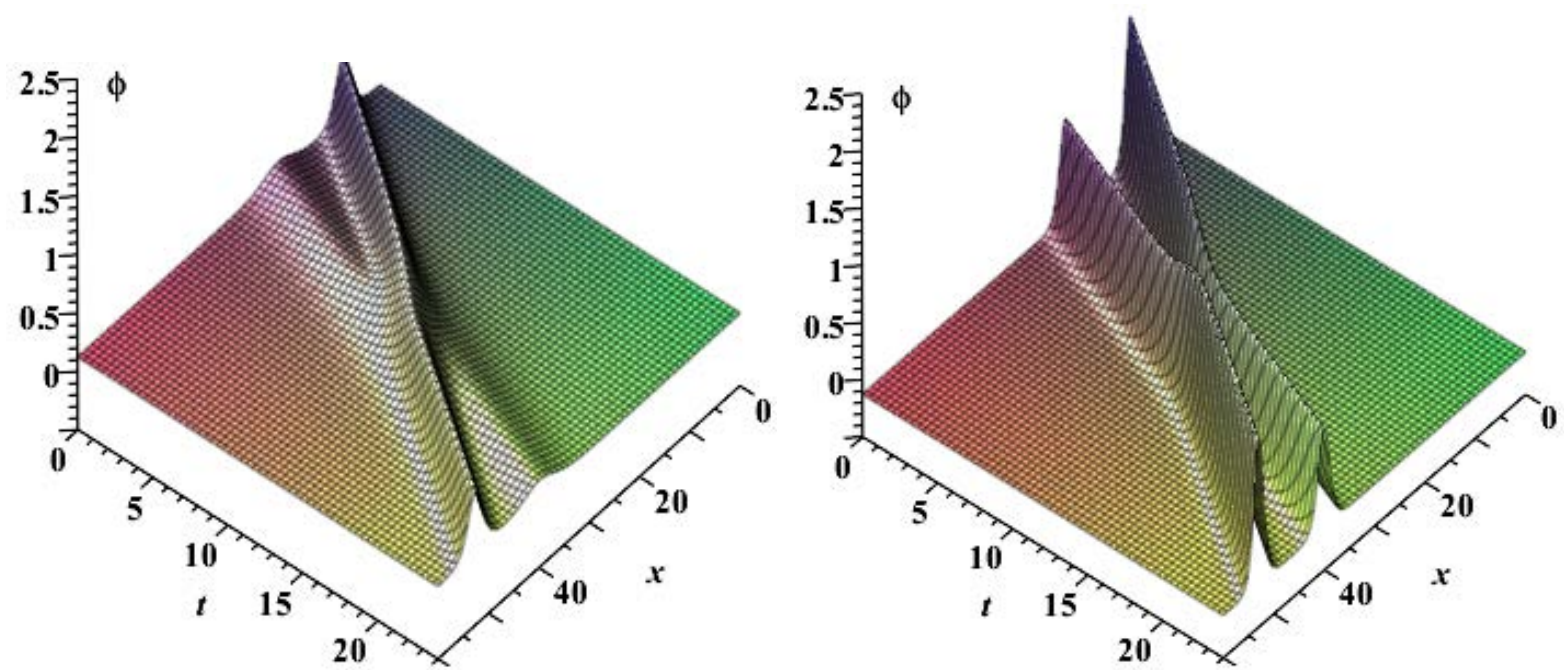

Figure 3. Spatiotemporal plots of the two-wave solution for $U=1.8, V=1, a=8.4, b=18, h=1 / 8$ (left) and $h=-1 / 8$ (right). 
The invariance of the ZHT structure with respect to differentiation and multiplication is shown by using 2d differentiation cascade of binomial structures and mathematical induction. Contrary to the asymmetric differentiation cascade in one dimension [18], the 2d differentiation cascade spreads in symmetric square waves. Compared with the $2 \mathrm{~d}$ series expansion, the ZHT structure considerably saves computational resources and simplifies results since it implies a low-order polynomial in one dimension and a series expansion in another dimension. The invariance of the ZHT structure enables other computational applications in nonlinear PDEs with solutions approaching a constantor vanishing at infinity.

The ZHT structural approximation and remainder are computed theoretically to any algebraic order. Summation of the two-wave solution in the invariant ZHT structures is implemented and presented both through regular and singular hyperbolic functions. When a reference level vanishes, the two-wave solution is reduced to the twosoliton solution. Negative reference levels considerably increase amplitudes and decrease dispersions of pulsatory waves compared with those for solitons with a vanishing reference level. The effects of positive reference levels are opposite, i.e. amplitudes of pulsatory waves are decreased and dispersions are increased.

\section{Acknowledgements}

The author thanks I. Tari for the stimulating discussion at the 2013 SIAM Annual Meeting. Support of the College of Mount Saint Vincent and CAAM is gratefully acknowledged.

\section{References}

[1] Zabusky, N.J. and Kruskal, M.D. (1965) Interaction of "Solitons" in a Collisionless Plasma and the Recurrence of Initial States. Physical Review Letters, 15, 240-243. http://dx.doi.org/10.1103/PhysRevLett.15.240

[2] Hirota, R. (1971) Exact Solutions of the Korteweg-de Vries Equation for Multiple Solitons. Physical Review Letters, 27, 1192-1194. http://dx.doi.org/10.1103/PhysRevLett.27.1192

[3] Drazin, P.G. (1983) Solitons. In: Reid, M., Ed., London Mathematical Society Lecture Note Series, No. 85, Cambridge University Press, Cambridge, 136.

[4] Izrar, B., Lusseyran, F. and Miroshnikov, V. (1995) Two-Level Solitary Waves as Generalized Solutions of the KdV Equation. Physics of Fluids, 7, 1056-1062. http://dx.doi.org/10.1063/1.868548

[5] Varley, E. and Seymour, B.R. (1998) A Simple Derivation of the N-Soliton Solutions to the Korteweg-de Vries Equation. SIAM Journal on Applied Mathematics, 58, 904-911. http://dx.doi.org/10.1137/S0036139996303270

[6] Vvedensky, D.D. (1992) Partial Differential Equations with Mathematica. Addison-Wesley Publishing Company, Wokingham.

[7] Miroshnikov, V.A. (1995) Solitary Wave on the Surface of a Shear Stream in Crossed Electric and Magnetic Fields: The Formation of a Single Vortex. Magnetohydrodynamics, 31, 149-165.

[8] Miroshnikov, V.A. (1996) The Finite-Amplitude Solitary Wave on a Stream with Linear Vorticity. European Journal of Mechanics, B/Fluids, 15, 395-411.

[9] Miroshnikov, V.A. (2002) The Boussinesq-Rayleigh Approximation for Rotational Solitary Waves on Shallow Water with Uniform Vorticity. Journal of Fluid Mechanics, 456, 1-32. http://dx.doi.org/10.1017/S0022112001007352

[10] Miroshnikovs, V. (1996) Coupled Solitary Waves in Viscous MHD and Geophysical Flows. Comptes Rendu Académie des Sciences Paris, 323, 23-30.

[11] Keller, J.B. (1948) The Solitary Wave and Periodic Waves in Shallow Water. Communications in Pure and Applied Mathematics, 1, 323-339.

[12] Laitone, E.V. (1960) The Second Approximation to Cnoidal and Solitary Waves. Journal of Fluid Mechanics, 9, 430444. http://dx.doi.org/10.1017/S0022112060001201

[13] Grimshaw, R. (1971) The Solitary Wave in Water of Variable Depth. Part 2. Journal of Fluid Mechanics, 46, 611-622. http://dx.doi.org/10.1017/S0022112071000739

[14] Longuet-Higgins, M.S. and Fenton, J.D. (1974) On the Mass, Momentum, Energy, and Circulation of a Solitary Wave II. Proceedings of the Royal Society A, 340, 471-493. http://dx.doi.org/10.1098/rspa.1974.0166

[15] Pennell, S.A. and Su, C.H. (1984) A Seventeenth-Order Series Expansion for the Solitary Wave. Journal of Fluid Mechanics, 149, 431-443. http://dx.doi.org/10.1017/S0022112084002731

[16] Pennell, S.A. (1987) On a Series Expansion for the Solitary Wave. Journal of Fluid Mechanics, 179, 557-561. http://dx.doi.org/10.1017/S0022112087001666 
[17] Miroshnikov, G.V. (2011) Hamiltonian Modeling of Pulsar Radiation Profiles. Far East Journal of Dynamical Systems, 17, 33-47.

[18] Miroshnikov, V.A. (2012) Dual Perturbations of the Poiseuille-Hagen Flow in Invariant Elliptic Structures. Advances and Applications in Fluid Dynamics, 11, 1-58.

[19] Schwenke, T. (1979) Sensitive Chaos: The Creation of Flowing Forms in Air and Water. Schocken Books, New York.

[20] Mollison, B. (1988) Permaculture: A Designer’s Manual. Tagari Publications, Tyalgum.

[21] Thompson, D.W. (1992) On Growth and Form. The Complete Revised Edition, Dover Publications, New York. 